This is the peer reviewed version of the following article: Doucerain, M. M., Deschênes, S. S., Gouin, J. P., Amiot, C. E., \& Ryder, A. G. (2017). Initial mainstream cultural orientations predict early social participation in the mainstream cultural group. Personality and Social Psychology Bulletin, 43(2), 245-258, which has been published in final form at $\backslash$ url $\{$ https://doi.org/10.1177/0146167216679642\}. Reuse is restricted to non-commercial and no derivative uses.

\title{
Initial Mainstream Cultural Orientations Predict Early Social Participation In The Mainstream CulturalGroup
}

\author{
Marina M. Doucerain ${ }^{1 *}$, Sonya S. Deschênes ${ }^{1}$, Jean-Philippe Gouin ${ }^{1}$, Catherine E. \\ Amiot $^{2}$, and Andrew G. Ryder ${ }^{1,3}$ \\ ${ }^{1}$ Concordia University (Montréal, Québec, Canada) \\ ${ }^{2}$ Université du Québec à Montréal \\ ${ }^{3}$ Jewish General Hospital (Montréal, Québec, Canada)
}

\begin{abstract}
This work adopts a perspective that construes acculturation as a dynamic intergroup process and social contact with members of the new community as a key mechanism underlying cultural adaptation. We argue that migrants' initial self-reported mainstream cultural orientation constitutes an important antecedent of early social participation in the new community. Results from two longitudinal studies of newly arrived international students $(N=98$ and $N=60)$ show that more positive initial mainstream cultural orientations prospectively predict higher social participation specifically in the mainstream group over the following months. This relation held after controlling for important alternative predictors, namely extraversion/shyness, mainstream language proficiency, and respiratory sinus arrhythmia, a physiological index of social engagement capacity. These studies focus on the very initial stages of the temporal dynamics of acculturation, contribute to bridging research on acculturation and on intergroup relations, and establish a link between cultural orientations, a subjective attitudinal construct, and concrete social engagement behaviors.
\end{abstract}

Keywords: cultural orientations, social participation, longitudinal, intergroup relations, acculturation, intergroup contact

\section{Introduction}

"Preferences are not competences", warns Boski (2008, p. 144) in his review of acculturation research. In a field where most studies focus on people's self-reported attitudes toward their various

\footnotetext{
* Correspondence concerning this article should be addressed to Marina M. Doucerain, 100 Sherbrooke Street West, Montréal, Québec, Canada H3C 3P8, doucerain.marina@uqam.ca or Andrew G. Ryder, Concordia University, 7141 Sherbrooke Street West, Montreal, Quebec, H4B 1R6. Contact: or andrew.ryder@concordia.ca
} 
cultural groups, this observation is particularly relevant. Considering that acculturation is a complex process of resocialization and culture acquisition, attitudinal scales can only capture one aspect of this complex and multifaceted experience. In addition, some critics have argued that elevated scores on acculturation scales might reflect more an appreciation of multiculturalism than actual cultural knowledge or competence (Boski, 2008). In other words, the relation between high scores on acculturation scales and concrete individual behaviors and cultural practices are unclear (Brown \& Zagefka, 2011).

As a step toward addressing this issue, the present work examines the role of cultural orientations - a subjective, attitudinal construct - as potential antecedents of early social engagement behaviors in migrants' cultural communities. We adopt the perspective that acculturation is to a large extent a dynamic intergroup process in which intergroup contact plays a key role (Brown \& Zagefka, 2011) and focus on the very initial stages of acculturation. We report on two longitudinal studies investigating the hypothesis that initial mainstream cultural orientation prospectively and specifically predicts contact with members of the mainstream group in the months following. Scores on cultural orientation scales may be limited in capturing the texture of migrants' lived experience or in directly assessing their competence in the new culture, but they might nonetheless represent an important predictor of social contact in the dominant cultural group, one of the behavioral cornerstones of acculturation.

\section{Acculturation: Culture Acquisition and the Role of Social Participation}

Psychological acculturation encompasses the change processes resulting from a person living in a new cultural environment (Sam \& Berry, 2010), including for example acquiring a new language, learning new social norms, forming new social relationships, and renegotiating social identities. At the core, most of these transformations hinge on acquiring the meanings and practices of a new cultural tradition. As such, acculturation is to a large degree a matter of second-culture acquisition (Rudmin, 2009). Although the exact mechanisms by which migrants develop cultural knowledge of their new context are largely unknown, researchers have argued that cultural transmission occurs through social interactions (Kashima, 2014). Through repeated interactions with members of the new cultural group, migrants construct and integrate a shared social reality that enables them to be functional members of that community (Wan, Dach-Gruschow, No, \& Hong, 2010; see also Kashima, 2014 for a similar account of cultural transmission outside of immigration contexts).

In parallel, researchers have underscored the relevance of theories of intergroup relations in acculturation research (Van Oudenhoven, Ward, \& Masgoret, 2006) and proposed that acculturation be considered as a dynamic intergroup process between immigrant and mainstream groups (Brown \& Zagefka, 2011). Numerous studies on intergroup relations have shown that intergroup contact - of which migrants' social participation in the mainstream group is a prime example - is crucial to improving intergroup relations (Pettigrew \& Tropp, 2006). Thus, an intergroup perspective on acculturation also highlights the centrality of migrants' social contact with members of the mainstream cultural group in acculturative processes. As a corollary, in order to facilitate the acculturation process, it is imperative to better understand what predicts migrants' social participation in the new community.

\section{Temporal Dynamics and the Importance of Initial Conditions}

Although acculturation is by definition a process of change over time, most studies on this subject are crosssectional. Fortunately, acculturation researchers are gradually taking heed of this issue and a number of longitudinal studies have been published in the last decade, with a general tendency to examine changes in adjustment and cultural orientations in youth (Doucerain, Segalowitz, \& Ryder, in press). Such studies contribute greatly to our understanding of the temporal dynamics of acculturation, but most of them include samples of migrants who have lived in the new country for varying numbers of years (with samples sometimes combining first- and second-generation immigrants: e.g., Brown et al., 2013) and therefore capture migrants at 
heterogeneous points along their acculturation trajectory. Very few studies have examined the very beginning of the acculturation process in "temporally homogeneous" samples, which is the focus of this work.

As in many transitions, initial conditions play a crucial role in influencing trajectories of change. Many important life transitions, such as immigration, are foreseen, leading individuals to engage in planning and anticipatory behaviors (Amiot, de la Sablonnière, Terry, \& Smith, 2007). Thus, people settle in a new country already equipped with predispositions that will influence their acculturation trajectory (Tartakovsky, 2009; Mähönen \& Jasinskaja-Lahti, 2012). Methodologically, the best design to examine the role of initial conditions in acculturation consists in identifying potential migrants in their country of origin and assessing constructs of interest shortly before migration. This approach presents considerable logistic difficulties, however, which probably explains why it is seldom employed. A more feasible alternative is to assess migrants upon or very shortly after migration. This is the approach employed here, with a focus on cultural orientations as an antecedent of social participation. We propose that migrants' initial self-reported cultural orientation toward the mainstream cultural group predicts social contact with members of that group. We now turn to how cultural orientations are conceptualized.

\section{Cultural Orientations: Definition and Conceptual Underpinnings}

Cultural orientations are characterized by considerable conceptual heterogeneity (Brown \& Zagefka, 2011; Rudmin, 2009), including attitudinal, motivational, behavioral, identity, or value components, depending on the definition used. Adding to this conceptual murkiness, terms are often used interchangeably (Liebkind, 2001). Here, we adopt a common and relatively broad conceptualization whereby cultural orientations reflect the extent to which migrants wish to maintain their culture of origin (heritage orientation) and, in parallel, wish to adopt the new culture (mainstream orientation; Bourhis, Moïse, Perreault \& Sénécal, 1997; Snauwaert et al., 2003). Thus, positive cultural orientations encompass not only migrants' desire for social contact with members of the heritage and mainstream groups, but also a positive outlook on these cultural traditions. In the case of recent migrants settling into a new cultural environment, the initial mainstream orientation reflects primarily a person's stance toward initiating and establishing engagement in the mainstream cultural group, with positive orientations indexing openness toward intergroup contact and cultural involvement.

In the dominant bidimensional acculturation framework, mainstream and heritage cultural orientations constitute two independent dimensions (Ryder, Alden, \& Paulhus, 2000). As many other researchers, we examine these cultural orientations directly, but others focus rather on acculturation strategies (integration, assimilation, separation, and marginalization; Berry, 2005), the fourfold typology resulting from crossing the mainstream and heritage orientations. Given our present emphasis on social participation in the mainstream group specifically, a dimensional approach, which examines the independent influence of a migrant's cultural traditions, is preferable over a typological approach, which considers the combined influence of two cultural traditions. Cultural orientations are a key construct in acculturation research, and are associated with a wide range of adjustment outcomes among migrants (Nguyen \& Benet-Martinez, 2013).

\section{Cultural Orientations as Predictors of Social Participation}

Migrants arrive in a new country with predispositions toward social contact and cultural engagement in the new community. Conceptually, it makes intuitive sense that greater initial openness toward establishing crosscultural relationships would predict greater actual intergroup contact. To the best of our knowledge, this longitudinal association between initial cultural orientations and ensuing mainstream social participation has not be investigated directly. Nonetheless, a number of studies on neighboring constructs can be brought to bear on this question. 
Because the mainstream cultural orientation encompasses a desire for social contact with members of the mainstream group, it is conceptually close to desired closeness or social distance aspects of intergroup attitudes. Consistent with the contact hypothesis (Allport, 1954), numerous studies have shown that greater intergroup contact is associated with more positive intergroup attitudes (Pettigrew \& Tropp, 2006). A few of these studies are longitudinal and have focused on immigration contexts (rather than on intergroup relations between longstanding groups, such as e.g., "Whites" and "Blacks" in the US, which is more typical in the intergroup literature). In addition to the assumed contact to attitudes effect, these studies have shown that intergroup attitudes predicted intergroup contact at later assessments, which is in line with the directionality of effect hypothesized here. In a large scale sample of ethnic minority students in the US, Levin and colleagues (Levin, van Laar, \& Sidanius, 2003) found that intergroup attitudes toward mainstream American in the first year of college were positively associated with intergroup contact in years two and three, which in turn positively predicted intergroup attitudes in the last year of college. Similarly, in a study of ethnic minority and majority groups in three European countries, Binder and colleagues (2009) showed that lesser desire for social distance at time 1 predicted greater intergroup contact at time 2, and vice-versa.

These authors raised the need to examine initial conditions of intergroup relations to disentangle the directionality of effects. Accordingly, Eller and Abrams (2003) found that American students newly arrived in Mexico who reported more desire for contact and interpersonal closeness with Mexicans shortly upon arrival also reported greater intergroup contact two weeks later. Although the very small sample size of this study limits confidence in these effects, they were later replicated in two other small longitudinal studies (Eller \& Abrams, 2004). Overall, the researchers found that the attitude to contact association was stronger than the reverse. As they note, "it makes intuitive sense that affect can be a precursor of intergroup friendship and even willingness to engage in more contact" (Eller \& Abrams, 2004, p. 240).

Outside of research on intergroup relations, studies examining cultural/ethnic identification among migrants are also relevant. Cultural orientations are different from cultural identity, but they are conceptually close, and as noted earlier, the two terms are often used interchangeably in acculturation research (Liebkind, 2001). Crosssectionally, Yip and Cross (2004) used a daily diary to show that Chinese-American youth who identified more strongly with their heritage cultural group showed more behavioral involvement in that community. Longitudinally, Stoessel, Titzmann, and Silbereisen (2012) found that stronger identification with the mainstream cultural group predicted greater social participation over time in that group, among adolescent diaspora immigrants to Germany and Israel. Finally, even outside of psychology, longitudinal research in demography using a large scale national survey showed that immigrants to Canada who rated the importance of Canadian traditions higher in the first year post-migration reported more numerous inter-ethnic friendships during the following three years (Martinovic, van Tubergen, \& Maas, 2011).

These studies did not directly investigate the relation between cultural orientations and social participation, and, with the exception of the Eller and Abrams 2003 study, they did not focus on the very beginning of the acculturation or intergroup process. However, taken together, these results support our overarching hypothesis that migrants' positive initial orientation toward the mainstream cultural group would prospectively predict higher social participation in that community in the months that follow.

\section{Study Design to Test the Directionality of Effects}

In their move to a new country, migrants bring along expectations and cultural orientations that reflect their pre-migration experience. The present work focuses on the role of these initial cultural orientations on migrants' patterns of social participation in the early stages of their acculturation process. We did not have access to participants pre-migration. Therefore, in order to demonstrate the temporal precedence of migrants' initial cultural orientations, we employ a longitudinal design where we assess the impact of cultural orientations endorsed very shortly after arrival in the new society (hereafter referred to as initial or baseline orientations) on later social participation. Migrants' attitudes toward heritage and mainstream cultural groups change over time as 
a result of various acculturative experiences. Therefore, it is important to assess cultural orientations at a time where they are still unshaped by these post-migration experiences. International students are well-suited for this approach as they represent a highly accessible population compared to other groups of recent migrants. In addition, their time of arrival is relatively predictable based on the academic year cycle, allowing us to recruit them almost immediately after settlement. We expect that baseline cultural orientations to the mainstream cultural group prospectively predict social participation in that group. As a stricter test of the role of initial cultural orientations, the reverse temporal model was also tested.

\section{Overview of the Studies}

Two longitudinal studies tested the overarching hypothesis that international students' initial cultural orientation to the mainstream group statistically predicts social participation in that group prospectively and specifically, above and beyond important alternative predictors. In Study 1, we examined the role of baseline mainstream cultural orientations in predicting friendships in the mainstream language. Study 2 replicated and extended the first study by further testing the specificity of the relation: international students' social participation was examined in several cultural groups and we controlled for extraversion and respiratory sinus arrhythmia (RSA), an important physiological marker of social engagement capacities. In the theoretical framework adopted here, acculturation to a large degree involves cultural acquisition through social interactions, and therefore rests heavily on intergroup contact. As such, the expected results would support the idea that initial cultural orientations are antecedents of a key mechanism underlying acculturative changes and fostering intergroup relations.

These studies make several important contributions. First, they contribute to the limited body of work on the temporal dynamics of acculturation. In addition, the focus on initial conditions complements existing longitudinal studies that seldom investigate this particular stage of acculturation. Second, by examining the relation between cultural orientations and intergroup contact, they bridge research in acculturation and in intergroup relations, two strands of research that are often kept separate in spite of their complementarity (Brown \& Zagefka, 2011; Van Oudenhoven et al., 2006). As such, this research also has the potential to enrich our understanding of intergroup relations by focusing on initial conditions of contact in a context less commonly studied. Finally, the current work can potentially shed some light on the relation between cultural orientations, a subjective attitudinal construct, and concrete behaviors, a relation which is unclear at best in acculturation research (Boski, 2008). Thus, we seek to put to the test the proposal that in the domain of acculturation, as in other domains, "there may be less than perfect correspondence between people's attitudes assessed in the abstract and how they actually behave in concrete situations" (Brown \& Zagefka, 2011, p. 172).

\section{Study 1}

The formation of new friendships in the new environment is beneficial for international students' adjustment (Hendrickson, Rosen, \& Aune, 2001), as well as an important marker of social participation in that environment. Cross-group friendships also represent a particularly beneficial form of intergroup contact (Pettigrew \& Tropp, 2006). This study examines the prospective role of initial mainstream cultural orientations in that process. Language proficiency is an important predictor of psychosocial adjustment among international students (Zhang \& Goodson, 2011) and social participation in the mainstream group hinges on the ability to communicate in the local language. Therefore, we control for proficiency in the mainstream language both in terms of design and 
analysis: to minimize the confounding factors of shared linguistic background, we selected only individuals whose native language was different from that of the mainstream cultural group and we statistically controlled for proficiency in the mainstream language. Shyness was also included as a covariate, as past research has shown that shyness, a stable individual difference in social withdrawal, negatively and prospectively predicts friendship formation (Asendorp \& Wilpers, 1998).

Our first hypothesis is: (H1) after controlling for shyness and proficiency in the mainstream language, there will be a positive and statistically significant relation between baseline cultural orientation toward the mainstream cultural group and number of cross-linguistic friendships in the new cultural environment over time . In line with the expected temporal precedence of cultural orientations, a secondary follow-up hypothesis is that (H2) the reverse temporal relation - more numerous initial friendships prospectively predicting more positive cultural orientation to the mainstream cultural group over time - will not be statistically significant.

\section{Methods}

Participants and procedure. Our sample comprised 98 newly arrived international students at universities in Montreal, Quebec, Canada (23 attending a French-speaking university and 75 attending an English-speaking university), a linguistically and culturally diverse city that includes Francophones and Anglophones as the two mainstream ethnolinguistic groups. In very broad cultural terms, Canada can be characterized as an individualistic cultural context (Hofstede, 2001). To ensure a valid measurement of baseline cultural orientations, participants were eligible only if they had arrived less than three months prior to the first assessment. In order to minimize the confounding impact of shared linguistic backgrounds, the present sample - drawn from a larger sample of new international students - included only people whose native language was different from the mainstream language of their university (i.e., different from French for participants attending a French-speaking university and different from English for those attending an English-speaking university). This ensured that friendships reported in this study would be formed across a linguistic divide for all participants. This criterion eliminated for example English-speaking American students forming new friendships in English at an Englishspeaking university, which is likely a qualitatively different phenomenon than friendship formation for students from e.g., China. Participants (51 females, $M_{\text {age }}=23.08$ years, $S D_{\text {age }}=4.55$ ) came from a variety of world regions: 35 from Europe, 13 from Africa, 15 from South America, 14 from South Asia, 10 from South-East Asia, 5 from the Middle East, and one from North America. Five participants did not provide information about their country of origin.

Our longitudinal design included four time points. The first assessment (T1) took place on average 27 days $(S D=18)$ after arrival. The second (T2), third (T3), and fourth (T4) measures occurred three, five, and seven months post-baseline, respectively. At each time point, participants were contacted via email and completed the surveys online. They received financial compensation for their time and the local Institutional Review Board of both universities approved the study. The total attrition rate was $29.59 \%\left(n_{\mathrm{T} 1}=98, n_{\mathrm{T} 2}=95, n_{\mathrm{T} 3}=67, n_{\mathrm{T} 4}=69\right)$.

\section{Measures.}

Social participation. Participants reported the number of friends with whom they interact in the mainstream language of their university (e.g., friendships in French if they attend a francophone university). This question ("How many friends do you have with whom you usually speak English/French") was administered at each time point and yielded our measure of social participation.

Cultural Orientations. The Quebec-specific version of the Vancouver Index of Acculturation (VIA, Ryder et al., 2000) is a 30-item questionnaire with three parallel subscales assessing orientations toward the heritage group (VIA-H), the mainstream French-Canadian group (VIA-FC), and the mainstream English-Canadian group (VIA-EC). Participants rated their agreement to items such as, "I am interested in having English-Canadian friends" on a 9-point rating scale, with higher scores indicating more agreement. Mainstream cultural orientation (VIA-M) was operationalized as scores on the VIA-FC subscale for participants attending a French-speaking 
university and as scores on the VIA-EC subscale for participants attending an English-speaking university. Past research has shown that the VIA is a valid and reliable cultural orientation questionnaire (Huynh, Howell, \& Benet-Martínez, 2009; Ryder et al., 2000). Internal consistency in this sample was acceptable for both mainstream (Cronbach's $\alpha_{\mathrm{T} 1}=.77$ for VIA-FC and $\alpha_{\mathrm{T} 1}=.76$ for VIA-EC) and heritage (Cronbach's $\alpha_{\mathrm{T} 1}=.91$ ) subscales. For the present purposes, baseline VIA-M and VIA-H scores were used as predictors. Time-varying VIA-M scores were used only for testing reverse temporal precedence in $\mathrm{H} 2$, and as an additional control in supplementary analyses.

Mainstream language proficiency. Four items (adapted from Segalowitz, 2009a) assessed participants' perceived ability to read, understand, write, and speak their university's dominant language on a 7-point rating scale ranging from "(1) Very poor" to "(7) Native-like." Internal consistency in this sample was very good (Cronbach's $\alpha=.92$ for French and $\alpha=.93$ for English).

Shyness. The Revised Cheek and Buss Shyness Scale (Cheek \& Briggs, 1990) is a 14-item questionnaire assessing shyness on a 5-point rating scale with higher scores indicating more agreement. A sample item is "I am socially somewhat awkward." Communicating and forming new friendships across cultural and linguistic divides can be challenging for international students (Williams \& Johnson, 2011), which may lead them to reinterpret how shy they are. Consequently, we used baseline perceptions of shyness in the heritage cultural group as a measure of trait shyness. Internal consistency in this sample was good (Cronbach's $\left.\alpha_{T 1}=0.84\right)$.

Analytic approach. The logarithmic transformation of our social participation variable was used in the analysis in order to normalize the distribution of residuals, which departed significantly from normality when using the raw variable. Indeed, a histogram showed that the raw variable was positively skewed with many participants reporting few friends. We used longitudinal mixed effects modeling to examine the role of initial cultural orientations in predicting social participation over time. To test H1, variables were entered hierarchically, with mainstream cultural orientations being entered last. All models specified a random effect for the intercept only and were fitted with restricted maximum likelihood (REML; Pinheiro \& Bates, 2000). Pseudo $R^{2}$ values were derived by comparing variance components (residual and intercept) of substantial models to the null model (Singer \& Willett, 2003). We also report Nakagawa and Schielzeth's $R^{2}$ values for fixed effects (Nakagawa \& Schielzeth, 2013). Likelihood ratio tests (LRT) used to compare model fit and confidence intervals were obtained using parametric bootstrapping (5000 samples). Given that the results of a priori power analysis to determine sample size are very sensitive to assumptions made and often impractical (Simonsohn, 2014), and given that post hoc power analysis has been described as fallacious (Kline, 2004), we rely preferentially on confidence intervals and effect sizes. Analyses were conducted with the lme 4 and pbkrtest packages in $R$ version 3.1.1. Missing data were handled by using restricted maximum likelihood estimation in all models. In terms of data preparation, univariate outliers were winsorized to three median absolute deviations around the median (Leys, Klein, Bernard, \& Licata, 2013). One participant was identified as a multivariate outlier (based on Mahalanobis distances evaluated at $p<.001$ ) and was therefore removed from the analysis (Myers, Gamst \& Guarino, 2013).

\section{Results and Discussion}

Descriptive results. Upon arrival in the new cultural context (i.e., baseline assessment), participants reported positive orientations toward their heritage cultural group, as measured by the VIA-H $(M=6.64$, $S D=1.41$ ), and fairly positive orientations toward the mainstream cultural group, as measured by the VIA-M $(M=5.78, S D=0.97)$. Overall, they reported high levels of mainstream language proficiency $(M=5.65, S D=1.02)$, indicating very good language proficiency for participants overall. As shown in Table 1 (model 1), social participation, as measured by the number of friends in the mainstream language (log-transformed), increased over time $(\beta(S E)=0.13(0.03), t(226.1)=4.46, p<.001,95 \% \mathrm{CI}=[0.07 ; 0.19])$. In other words, the number of mainstream friends increased by $2 \%$ on average for every 10 days lived in the new country. 
Table 1 | Study 1: Modeling Changes in Number of Friends in the Mainstream Language

\begin{tabular}{|c|c|c|c|c|}
\hline & Model 1 & Model 2 & Model 3 & Model 4 \\
\hline Intercept & $1.98(0.09)^{\star * \star}$ & $2.11(0.60)^{\star * *}$ & $2.16(0.73)^{\star *}$ & $1.62(0.75)^{\star}$ \\
\hline Time $^{a}$ & $0.02(0.00)^{* * *}$ & $0.02(0.00)^{* * *}$ & $0.02(0.00)^{* * *}$ & $0.02(0.00)^{* * *}$ \\
\hline Baseline shyness & & $-0.30(0.17)^{\dagger}$ & $-0.30(0.17)^{\dagger}$ & $-0.34(0.17)^{*}$ \\
\hline Language proficiency & & $0.12(0.06)^{\dagger}$ & $0.12(0.06)^{\dagger}$ & $0.10(0.06)$ \\
\hline Baseline VIA-H & & & $-0.01(0.06)$ & $-0.09(0.07)$ \\
\hline Baseline VIA-M & & & & $0.23(0.09)^{*}$ \\
\hline Residual pseudo- $R^{2}$ & .07 & .04 & .04 & .05 \\
\hline Intercept pseudo- $R^{2}$ & .02 & .12 & .11 & .15 \\
\hline$R^{2}$ fixed effects & .02 & .06 & .06 & .11 \\
\hline$A I C^{b}$ & 626.08 & 610.37 & 612.36 & 608.37 \\
\hline $\mathrm{BIC}$ & 641.09 & 632.73 & 638.45 & 638.18 \\
\hline LRT(df) & $17.18(1)^{* * *}$ & $6.46(2)^{*}$ & $0.00(1)$ & $5.96(2)^{*}$ \\
\hline
\end{tabular}

Note. Entries represent unstandardized coefficients (SE). ${ }^{\dagger} p<.10,{ }^{*} p<.05,{ }^{* *} p<.01,{ }^{* * *} p<.00 .1$ aThe time unit used corresponds to 10 days. b Models were refitted using maximum likelihood to compute AIC and BIC values.

Hypothesis Testing. Table 1 presents results of the mixed-effects modeling of friendships in the mainstream language over time. Controlling for self-reported mainstream language proficiency, baseline shyness, and heritage cultural orientation, the positive association between initial mainstream cultural orientations and social participation (model 4) was statistically significant $(\beta(S E)=0.28(0.11), t(90.75)=2.42, p=.01,95 \% \mathrm{CI}=$ $[0.05 ; 0.49]$ ), thus supporting H1. This indicates that a one-unit increase in baseline VIA-M scores was associated with a $26 \%$ increase in the number of mainstream friends. Moreover, VIA-M scores were the strongest predictor of social participation in the mainstream ethnolinguistic group in the final model ( $\beta=.28$, compared to -.17 for shyness and .12 for language proficiency). The introduction of baseline VIA-M scores (model 4) accounted for an additional 4\% in intercept variance and increased $R^{2}$ for fixed effects from .06 to .11 . In addition, providing some evidence of the cultural specificity of the relation between cultural orientations and social participation, the heritage orientation, as measured by VIA-H scores, did not predict friendships in the mainstream language $(\beta(S E)=-0.14(0.11), t(88.92)=-1.32, p=.19,95 \% \mathrm{CI}=[-0.35 ; 0.07])$, and introducing VIA-H as a predictor (model 3 ) did not account for any additional variance.

Supporting H2, the same model with reverse temporal order revealed that initial friendships in the mainstream language was not a statistically significant predictor of mainstream cultural orientations scores over time $(\beta(S E)=0.12(0.08), t(91.13)=1.53, p=.13,95 \% \mathrm{CI}=[-0.04 ; 0.28])$. Thus, the results support both hypotheses: controlling for mainstream language proficiency and baseline shyness, a more positive cultural orientation toward the mainstream cultural group prospectively predicts more numerous friendships in that group over the following months, but the reverse temporal relationship was not statistically significant.

Supplementary analyses. To further establish the role of baseline mainstream cultural orientation as a 
predictor of mainstream social participation, we also tested the same models dropping Time 1 for all variables except for mainstream cultural orientation, our key predictor. This establishes a lag between mainstream cultural orientation and social participation (and other covariates) and therefore addresses potential concerns about synchronous effects. The pattern of results remained the same. Controlling for mainstream language proficiency, shyness, and heritage cultural orientation, the relation between baseline mainstream cultural orientations and social participation at subsequent assessments was statistically significant $(\beta(S E)=0.28(0.12), t(86.4)=2.26, p$ $=.03,95 \% \mathrm{CI}=[0.04 ; 0.52])$, whereas the reverse temporal relation between baseline social participation and mainstream cultural orientation at later assessments was not $(\beta(S E)=0.10(0.10), t(94.05)=0.99, p=.33,95 \%$ $\mathrm{CI}=[-0.09 ; 0.29])$. These supplementary results are also in line with both hypotheses.

Baseline VIA-M scores and VIA-M scores at later assessments are strongly correlated $(r=.55)$. Nevertheless, to further test the specific role of baseline cultural orientation over and beyond synchronous effects, we added time varying VIA-M scores as an additional control. The coefficient for baseline cultural orientation remained statistically significant $(\beta(S E)=0.25(0.12), t(88.80)=2.03, p=.046,95 \% \mathrm{CI}=[.01$; $0.49])$, whereas the coefficient for time varying VIA-M scores was not $(\beta(S E)=0.08(0.06), t(176.51)=1.39, p$ $=.17,95 \% \mathrm{CI}=[-0.04 ; 0.19])$. These results support the hypothesis that the relation between baseline cultural orientation and social participation over the following months is specific and prospective.

\section{Study 2}

Study 2 replicates and expands on Study 1 by addressing several limitations. First, the measure used in study assessed "friendship". However, the meaning of friendship can vary widely not only between individuals, but also between cultural contexts (Baumgarte, 2013), which may bias the number of friends reported. Therefore Study 2 relies on a more objective measure of social participation: namely, the number of interlocutors with whom participants interact on a regular basis. Second, in Study 1 social participation was defined in language terms - friends with whom they interact in English or in French - and this definition raises the issue of the cultural specificity of the relation between cultural orientations, which are defined in cultural terms, and social participation. For example, a participant at a Francophone university may use French to interact not only with French-Canadians, but also with other international students. Critics of self-reported acculturation attitudinal scales have argued that high scores on these scales may reflect a general preference for multiculturalism (Boski, 2008), and therefore it is important to show that a positive orientation toward a cultural group is associated with social participation in that group specifically, and not in others. To address this issue, social participation in Study 2 is defined in cultural terms and social participation in three different groups is considered: the mainstream cultural group, the heritage cultural group, and other international students from a different cultural background.

Finally, although we controlled for individual differences in shyness, it is possible that the changes in social participation observed in Study 1 largely reflect a physiological proneness to form social relationships. To help rule out this alternative, Study 2 includes extraversion and respiratory sinus arrhythmia (RSA), a physiological index of social engagement capacity (Porges, 2007), as a more stringent control. The Polyvagal Theory suggests that through mammalian evolution, RSA became an index of a neurophysiological system enabling individuals to flexibly and rapidly regulate their physiology to facilitate activation of the social engagement system, which comprises a suite of behaviors related to navigating one's social environment (Porges, 2007). Indeed, the brain stem nuclei regulating RSA are sites of integration of information from the viscera and higher brain structures that modulate both cardiac activity and a collection of facial muscles implicated in emotional expression and social behavior. In accordance with the Polyvagal Theory, a number of studies have established an association between RSA and various facets of social functioning (e.g., Geisler, Kubiak, Siewert, \& Weber, 2013; Gyurak \& Ayduk, 2008; Smith et al., 2011). Upon settlement, international students face the important task of forming social relationships, complicated by the necessity to cross a cultural divide. Indeed, past research has highlighted the difficulties of forming cross-cultural friendships (Williams \& Johnson, 2011). As such, efficient recruitment 
and regulation of one's social engagement system, indexed by higher RSA, is likely to facilitate social participation in the new cultural group. This role of RSA in predicting social participation affords a further test of the specificity of the association between cultural orientations and social participation.

The first two hypotheses for Study 2 replicate those from Study 1 (reflecting changes in the controls included in this study). A third hypothesis targets the issue of cultural specificity, as follows: (H3a) Baseline cultural orientations to the mainstream cultural group are not statistically associated with social participation in the heritage cultural group or among international students from a different cultural background. Conversely: (H3b) Baseline cultural orientations to the heritage cultural group are not statistically associated with social participation in the mainstream cultural group or among international students from a different cultural background. We form no specific hypothesis regarding the relation between heritage cultural orientation and participation in the heritage cultural group because participation in that group is largely constrained by the demographic concentration of co-nationals in the settlement area. For example, a Chinese immigrant to Vancouver would have access to a larger heritage cultural group than a Gabonese immigrant. As such, social participation in that group is likely to reflect factors mostly outside of migrants' heritage cultural orientation.

\section{Methods}

Participants and procedure. Our sample comprised 60 newly arrived international students at an Englishspeaking university in Montreal, Quebec, Canada (29 females, $M_{\text {age }}=23.8$ years, $S D_{\text {age }}=3.49$ ). Participants came from a variety of ethnocultural backgrounds: 12 self-identified as Caucasians (e.g., American, Italian), 19 as East/South East Asians (e.g., Chinese, Korean), 13 as South Asians (e.g., Indian), 6 as Middle Easterners (e.g., Iranian), 5 as Latinos (e.g., Venezuelan), and one as Black (e.g., Nigerian). To ensure a valid measurement of baseline cultural orientations, participants were eligible only if they had arrived less than one month prior to the first assessment. In order to maximize homogeneity in terms of initial social functioning within the sample, the following inclusion criteria were applied: (1) having no friends or relatives residing in the new city prior to the participant's own arrival; (2) not being involved in a romantic relationship; (3) having migrated alone; (4) being committed to staying in the new country for at least two years; and (5) having received a score of at least 600 on the Test of English as Foreign Language (TOEFL). In addition, only people having no chronic health problems and not taking any prescribed medications regularly were included in the sample.

Our longitudinal design included three time points. The first assessment (T1) took place an average of 22 days $(S D=9.4)$ after arrival. The second (T2) and third (T3) visits occurred two and five months post-baseline, respectively. Each visit occurred between 9:30 am and 12:30 pm to limit the influence of diurnal variation on RSA (Bonnemeier et al., 2003). In addition, participants were asked to abstain from eating, consuming caffeine, smoking, and exercising for at least two hours prior to each assessment (Berntson et al., 1997). Participants received financial compensation for their time and the local Institutional Review Board approved the study. The total attrition rate was $8.33 \%\left(n_{\mathrm{T} 1}=60, n_{\mathrm{T} 2}=57, n_{\mathrm{T} 3}=55\right)$; only one participant missed both $\mathrm{T} 2$ and $\mathrm{T} 3$.

\section{Measures.}

Social participation. Participants reported the number of (a) Canadians, (b) international students from a different cultural background, and (c) persons from the same cultural background that they talk to regularly, i.e., at least once a week. Each number represented social participation in that specific group. These questions were adapted from Cohen, Doyle, Skoner, Rabin, and Gwaltney (1997), and Hendrickson et al. (2011). They were administered at each time point.

Cultural Orientations. Like in Study 1, the Vancouver Index of Acculturation (VIA, Ryder et al., 2000) was used. In Study 1, the correlation between VIA-FC and VIA-EC scores at T1 was very high $(r=.73)$, suggesting that the participants in this sample may not have yet realized the differences between these two groups at that point. Therefore, in this sample we used the heritage subscale (VIA-H) and a generic mainstream subscale assessing participants' orientations toward Canadians (VIA-M). Internal consistency in this sample was 
acceptable for both mainstream (Cronbach's $\left.\alpha_{\mathrm{T} 1}=.76\right)$ and heritage (Cronbach's $\alpha_{\mathrm{T} 1}=.80$ ) subscales. Like in Study 1, baseline VIA-M and VIA-H scores were used as predictors. Time-varying VIA-M scores were used only for testing reverse temporal precedence in $\mathrm{H} 2$, and as an additional control in supplementary analyses.

Respiratory Sinus Arrhythmia (RSA). Participants were fitted with a chest band hardwired with a digital inter-beat interval recorder (Polar RS800CX). They were instructed to sit alone in a quiet room and to breathe normally while relaxing as much as possible during the 15 -minute recording. Cardiac inter-beat intervals were recorded continuously, using a sampling rate of 1000 samples per second. In order to minimize the impact of the novelty response to the laboratory environment, the first 5 minutes of the recording periods were excluded from analysis. Recording artifacts were manually identified and corrected using the CardioEdit software (2007). Fewer than $2 \%$ of the data points were edited across the three visits. RSA was computed with the Cardiobatch (2007) software using the Porges and Bohrer's (1990) moving polynomial approach.

English proficiency. We used the same items than in Study 1. Internal consistency in this sample was very good (Cronbach's $\alpha=.94)$.

Extraversion. We used extraversion instead of shyness as an alternative measure of sociability. The extraversion subscale of the Eysenck Personality Questionnaire Brief Version (EPQ-BV; Sato, 2005) is a 12-item questionnaire assessing extraversion on a 5-point rating scale. A sample item is: "Do you usually take the initiative in making new friends?" Total scores can range from 1 to 5 . Internal consistency in this sample was acceptable (Cronbach's $\alpha=.74)$.

Analytic approach. We used the same analytic approach than in Study 1, with one difference: we used multivariate longitudinal mixed effects modeling in order to examine the covariation of social participation in the three cultural groups within a single model.

\section{Results and Discussion}

Descriptive results. On average, across the three time points, participants interacted weekly with six persons from the same cultural heritage $(M=5.96, S D=3.47)$, six international students with different cultural origins $(M=5.50, S D=3.57)$, and four Canadians $(M=4.48, S D=3.47)$. As shown in Table 2 (model 1), social participation increased over time in all groups (Canadian group: $\beta(S E)=0.12(0.05), t(339.26)=2.43, p=.03$, $95 \% \mathrm{CI}=[0.02 ; 0.22]$; International students group: $\beta(S E)=0.12(0.05), t(339.64)=2.36, p=.02,95 \% \mathrm{CI}=$ $[0.02$; 0.21]; Heritage group: $\beta(S E)=0.16(0.05), t(339.93)=3.16, p=.002,95 \% \mathrm{CI}=[0.06 ; 0.26])$.

Upon arrival in the new cultural context (i.e., baseline assessment), participants reported positive orientations toward the heritage cultural group, as measured by the VIA-H $(M=6.54, S D=0.99)$, and fairly positive orientations toward Canadians, as measured by the VIA-M $(M=5.73, S D=0.91)$. Overall, they reported high levels of English proficiency $(M=5.44, S D=1.11)$, indicating very good English proficiency for participants overall.

Higher overall time-varying RSA was associated with greater social contact in the mainstream cultural group (in model $2, \beta(S E)=0.20(0.08), t(215.69)=2.52, p=.01,95 \% \mathrm{CI}=[0.04 ; 0.36])$ and greater social contact with international students from different cultural origins $(\beta(S E)=0.15(0.08), t(200.16)=1.96, p=.05$, $95 \% \mathrm{CI}=[0.00 ; 0.33])$. However, RSA did not predict social contact with members of participants' own cultural group $(\beta(S E)=-0.02(0.08), t(226.84)=-0.25, p=.80,95 \% \mathrm{CI}=[-0.18 ; 0.14])$.

Hypothesis testing. Table 2 presents results of the multivariate multilevel modeling of number of interlocutors over time. Controlling for RSA, self-reported English proficiency, extraversion, and heritage orientations, the positive association between initial mainstream cultural orientations and social contact with Canadians was statistically significant (model $5 \beta(S E)=0.25(0.11), t(54.30)=2.28, p=.03,95 \% \mathrm{CI}=[0.04$; 0.46]), thus supporting H1. In addition, the introduction of VIA-M scores accounted for an additional $8 \%$ in intercept variance in participation among Canadians. We tested the reverse temporal order, with mainstream 
cultural orientation over time as a the dependent variable. Consistent with H2, baseline number of Canadian interlocutors was not statistically associated with mainstream cultural orientation $(\beta(S E)=0.10(0.12), t(56.36)=$ $0.84, p=.40,95 \% \mathrm{CI}=[-0.15 ; 0.33])$.

Table 2 | Study 2: Multivariate Modeling of Changes in Number of Regular Interlocutors in the Different Cultural Groups

\begin{tabular}{|c|c|c|c|c|c|}
\hline & Model 1 & Model 2 & Model 3 & Model 4 & Model 5 \\
\hline \multicolumn{6}{|l|}{ Intercepts } \\
\hline International students & $4.77(0.55)^{\star \star *}$ & $-6.97(3.29)^{*}$ & $-8.61(4.17)^{*}$ & $-7.65(4.30)^{\dagger}$ & $-7.65(4.30)^{\dagger}$ \\
\hline Heritage group & $5.05(0.53)^{\star * *}$ & $6.39(3.45)^{\dagger}$ & $2.70(4.35)$ & $2.85(4.52)$ & $2.85(4.52)$ \\
\hline Canadians & $3.76(0.53)^{\star \star *}$ & $-6.77(3.25)^{*}$ & $-9.77(4.07)^{*}$ & $-9.77(4.07)^{*}$ & $-12.08(4.04)^{\star *}$ \\
\hline \multicolumn{6}{|l|}{ Time $e^{a}$} \\
\hline International students & $0.09(0.04)^{*}$ & $0.10(0.04)^{*}$ & $0.10(0.04)^{\star}$ & $0.10(0.04)^{*}$ & $0.10(0.04)^{*}$ \\
\hline Heritage group & $0.12(0.04)^{\star \star}$ & $0.11(0.04)^{\star *}$ & $0.11(0.04)^{\star *}$ & $0.11(0.04)^{* *}$ & $0.11(0.04)^{\star *}$ \\
\hline Canadians & $0.09(0.04)^{*}$ & $0.10(0.04)^{*}$ & $0.10(0.04)^{*}$ & $0.10(0.04)^{*}$ & $0.10(0.04)^{*}$ \\
\hline \multicolumn{6}{|l|}{ RSA } \\
\hline International students & & $0.64(0.32)^{\star}$ & $0.63(0.32)^{\dagger}$ & $0.64(0.32)^{*}$ & $0.64(0.32)^{\star}$ \\
\hline Heritage group & & $-0.08(0.33)$ & $-0.09(0.33)$ & $-0.09(0.33)$ & $-0.09(0.33)$ \\
\hline Canadians & & $0.81(0.32)^{*}$ & $0.81(0.32)^{*}$ & $0.81(0.32)^{*}$ & $0.81(0.31)^{\star}$ \\
\hline \multicolumn{6}{|l|}{ Extraversion } \\
\hline International students & & $-0.01(0.62)$ & $-0.05(0.62)$ & $0.07(0.63)$ & $0.07(0.63)$ \\
\hline Heritage group & & $1.01(0.64)$ & $0.93(0.64)$ & $0.95(0.66)$ & $0.95(0.66)$ \\
\hline Canadians & & $0.21(0.61)$ & $0.15(0.61)$ & $0.15(0.61)$ & $-0.15(0.61)$ \\
\hline \multicolumn{6}{|l|}{ English proficiency } \\
\hline International students & & $1.37(0.38)^{\star *}$ & $1.37(0.38)^{\star *}$ & $1.44(0.39)^{* * *}$ & $1.44(0.39)^{\star * \star}$ \\
\hline Heritage group & & $-0.74(0.40)^{\dagger}$ & $-0.73(0.40)^{\dagger}$ & $-0.72(0.41)^{\dagger}$ & $-0.72(0.41)^{\dagger}$ \\
\hline Canadians & & $0.80(0.37)^{\star}$ & $0.80(0.37)^{*}$ & $0.80(0.37)^{*}$ & $0.64(0.36)^{\dagger}$ \\
\hline \multicolumn{6}{|l|}{ Baseline VIA-H } \\
\hline International students & & & $0.27(0.42)$ & $0.41(0.44)$ & $0.41(0.44)$ \\
\hline Heritage group & & & $0.60(0.44)$ & $0.63(0.47)$ & $0.63(0.47)$ \\
\hline Canadians & & & $0.49(0.41)$ & $0.49(0.41)$ & $0.17(0.41)$ \\
\hline \multicolumn{6}{|l|}{ Baseline VIA-M } \\
\hline International students & & & & $-0.46(0.50)$ & $-0.46(0.50)$ \\
\hline Heritage group & & & & $-0.08(0.53)$ & $-0.08(0.53)$ \\
\hline Canadians & & & & & $1.08(0.47)^{\star}$ \\
\hline Residual pseudo- $R^{2}$ & .04 & .04 & .04 & .04 & .04 \\
\hline \multicolumn{6}{|l|}{ Intercept pseudo- $R^{2}$} \\
\hline International students & .00 & .28 & .26 & .27 & .27 \\
\hline Heritage group & .02 & .07 & .06 & .06 & .07 \\
\hline Canadians & .01 & .24 & .25 & .25 & .33 \\
\hline $\mathrm{AIC}^{\mathrm{b}}$ & 2757.3 & 2738.0 & 2740.0 & 2743.0 & 2739.5 \\
\hline $\mathrm{BIC}$ & 2799.8 & 2818.7 & 2833.4 & 2844.9 & 2845.7 \\
\hline LRT(df) & $21.05(3)^{\star * *}$ & $37.28(9)^{* * *}$ & $3.91(3)$ & $0.87(2)$ & \\
\hline
\end{tabular}

Note. Entries represent unstandardized coefficients (SE). ${ }^{\dagger} p<.10,{ }^{*} p<.05,{ }^{* *} p<.01,{ }^{* * *} p<.001$. ${ }^{\text {aT }}$ The time unit used corresponds to 10 days. ${ }^{b}$ Models were refitted using maximum likelihood to compute AIC and BIC values. 
Cultural specificity. Table 2 also presents results pertaining to cultural specificity. In line with hypothesis $\mathrm{H} 3 \mathrm{a}$, baseline VIA-M scores statistically predicted social participation neither among other international students $($ model $5 \beta(S E)=-0.10(0.11), t(57.60)=-0.93, p=.36(95 \% \mathrm{CI}=[-0.30 ; 0.10])$, nor in the heritage cultural group $(\beta(S E)=-0.02(0.12), t(57.60)=-0.14, p=.89,95 \% \mathrm{CI}=[-0.24 ; 0.21])$. Likewise, supporting hypothesis $\mathrm{H} 3 \mathrm{~b}$, baseline VIA-H scores, reflecting the heritage cultural orientation, were associated with number of regular interlocutors neither in the mainstream cultural group (model $5 \beta(S E)=0.04(0.10), t(53.30)=0.42, p=.67$ $(95 \% \mathrm{CI}=[-0.15 ; 0.24])$, nor among international students from a different cultural background (model $5 \beta(S E)$ $=0.09(0.10), t(56.60)=0.92, p=.36(95 \% \mathrm{CI}=[-0.10 ; 0.29])$. Notably, VIA-H scores did not predict the number of interlocutors in participants' own cultural group (model $5 \beta(S E)=0.15(0.11), t(56.40)=1.34, p=.19$ $(95 \% \mathrm{CI}=[-0.06 ; 0.36])$. Taken together, these results fully support our hypothesis regarding the cultural specificity of the relation between baseline cultural orientations to the mainstream cultural group and social participation in that group, as well as our other hypotheses.

Supplementary analyses. As in study 1, we tested a model establishing a lag between mainstream cultural orientation and social participation (and other covariates) by dropping Time 1 social participation variables. Even in this restrictive model with only two times points left, the pattern of results remained the same. Taking into account the same covariates as above, the relation between baseline mainstream cultural orientations and social participation at later assessments was statistically significant $(\beta(S E)=0.28(0.12), t(55.85)=2.35, p=.02$, $95 \% \mathrm{CI}=[0.04 ; 0.52])$, whereas the reverse temporal relation between baseline social mainstream participation and mainstream cultural orientation at subsequent assessments was not $(\beta(S E)=.12(0.13), t(53.37)=0.92, p=$. $36,95 \% \mathrm{CI}=[-0.14 ; 0.38])$. These supplementary results are also in line with both hypotheses.

Baseline VIA-M scores and VIA-M scores at later assessments were even more strongly correlated $(r=.78)$ than in Study 1, raising concerns about collinearity. Nevertheless, as in Study 1, the pattern of results remained the same in supplementary analyses where we added time varying VIA-M scores as an additional control (coefficient for baseline VIA-M: $\beta(S E)=.28(0.14), t(78.11)=1.96, p=.054,95 \% \mathrm{CI}=[0.003 ; 0.55]$;

coefficient for time varying VIA-M: $\beta(S E)=.01(0.12), t(170.04)=0.08, p=.93,95 \% \mathrm{CI}=[-0.22 ; 0.24])$. These results support the hypothesis that the relation between baseline cultural orientation and social participation over the following months is specific and prospective.

\section{General Discussion}

Critics of self-reported cultural orientations contend that these orientations are limited in furthering our understanding of migrants' adaptation processes (Chirkov, 2009). In response to this contention, this article proposed that cultural orientations are an important antecedent of actual social participation, conceptualized through intergroup contact and a key behavioral mechanism underlying acculturation processes and facilitating intergroup relations. The results from two longitudinal studies show that a more positive orientation toward the mainstream cultural group upon arrival is related to greater early social participation in that group, fully supporting the hypotheses. Specifically, the association between mainstream cultural orientation and mainstream social participation (1) meets temporal precedence criteria, (2) holds after controlling for important alternative predictors and is of appreciable magnitude, (3) shows cultural specificity, and (4) is replicated across two different measures of social contact. We do not claim that a positive mainstream cultural orientation causes mainstream social contact, but collectively these results support the plausibility of this direction of causation between these variables.

Future work should examine the factors moderating this relation as well as the boundary conditions constraining it. Research on acculturation has yielded a wealth of pertinent results. For example, it is likely that orientations of the receiving society toward migrants, as well as the larger sociopolitical climate, constrain how much social participation is possible in the mainstream community (see Bourhis et al., 1997 for a discussion of the interplay between migrants' and mainstream members' orientations). Similarly, perceived discrimination in 
migrants' local context may moderate the influence of mainstream cultural orientations on early social participation: migrants who arrive in the new country highly motivated to form social relationships with members of the mainstream group may struggle to do so in the face of high levels of discrimination. Accordingly, Titzmann, Silbereisen, \& Schmitt-Rodermund (2007) found that greater perceived discrimination predicted higher levels of intra-cultural friendships among adolescent immigrants to Germany. Given the cultural homogeneity in our samples, participants may have faced various degrees of discrimination. For the sake of parsimony, we narrowed our investigation to social contact and constructs most closely associated to it, but it would be important for future work to address this limited focus and consider the role of perceived discrimination.

Language proficiency is another important factor that might influence the relation between cultural orientations and social participation. Social interactions hinge on migrants' ability to communicate in the mainstream language. Language skills afford or prevent social participation in a way that is partly independent from cultural orientations. Past research has established some connections between language proficiency and community engagement among migrants (Chiswick \& Miller, 1996). Hence, the relation between cultural orientations and social participation may be subject to a minimum threshold of language proficiency. A strength of the present work was that our samples included only individuals with a functional level of English or French proficiency (participants attended university in English or French and reported high levels of proficiency in that language overall). As well, the sample in study 1 excluded native speakers of the mainstream language, thus addressing threshold (or ceiling) issues and allowing us to test the specific impact of cultural orientations on social participation. However, in future research it would be worthwhile to further explore the role of language as a gate keeping mechanism.

The results of Study 2 suggest that RSA might also play an important role in migration processes. Higher RSA at the first assessment was associated with greater social contact with members of the mainstream culture and with other international students, but not with members of participants' own cultural group. These findings not only provide further evidence of RSA as a physiological index of social functioning (Porges, 2007) but also suggest that individual differences in RSA predict adaptive social behaviors in a new cultural environment among recently migrated individuals. One possibility is that greater RSA is associated with greater ability to meet the sociopragmatic demands of cross-cultural communication. Successful communication between two interlocutors depends not only on linguistic skills (i.e., the proper use of pronunciation, syntax and vocabulary), but also on cultural scripts guiding people's behaviours and expectations (Schank \& Abelson, 1977) as well as sociopragmatic aspects of communication (Ranney. 1992). In the case of newly arrived migrants, the absence of these cultural scripts renders cross-cultural communication difficult (Nishida, 1999); nonetheless, higher social engagement capacities, indexed by higher RSA, may plausibly promote greater attunement to socio-pragmatic demands of cross-cultural communication, thus compensating for underdeveloped cultural scripts. This mechanism would explain the finding that RSA was not associated with social participation within participants' own cultural group, where they can rely on cultural scripts to guide social interactions. In line with this idea, Porter (2003) reported a positive relation between infants' RSA and the symmetrical co-regulation of communication in the mother-infant dyad. In a related vein, lower RSA was associated with greater pragmatic language impairment among children with Autism Syndrome Disorder (Klusek, Martin, \& Losh, 2013). Future research could explore further the possible relation between RSA and pragmatic communication abilities, a potentially important antecedent of social participation.

At a conceptual level, we can envision the relation between cultural orientations and social participation more broadly and consider the mechanisms that underlie it. Three different perspectives, corresponding to different levels of analysis and time scales are plausible - not only an attitudinal meso-level traditionally considered by acculturation researchers', but also an interactional micro-level, and a developmental macro-level. At the meso-level, initial cultural orientations are likely to direct migrants' attention and efforts in certain directions, thus influencing the likelihood of specific types of social interactions. For example, a Chinese migrant to Canada very open toward contact with Canadians might decide to do his groceries in a mainstream 
supermarket rather than an ethnic shop, or to choose an apartment in a mainly English-speaking neighborhood rather than a Cantonese enclave. This aggregation of small decisions increases the probability of daily interaction with Canadians. In this view, cultural orientations, reflecting individual differences in the degree of openness toward intercultural contact, motivate a pattern of preferential choices that accumulate to influence the likelihood of social participation in the mainstream community over time. In other words, cultural orientations have the potential to shape the local cultural ecology within which interactions will take place (Segalowitz, Gatbonton, \& Trofimovich, 2009).

The micro-level perspective takes place on a very short time scale and considers the momentary interaction as a unit of analysis: how orientations shape interactions between a migrant and a member of the mainstream group in the moment. For example, orientations may influence the extent to which migrants are motivated to work on establishing a common ground with their mainstream interlocutor in spite of potential cross-cultural difficulties, or the extent to which they will persist in the interaction in spite of perceived discrimination (provided of course sufficient language skills and confidence to successfully manage the interaction). More precisely, cultural orientations might influence interactions in the moment through the adoption of specific sociolinguistic markers indexing their social position (Pavlenko, 2004). Similarly, cultural orientations may influence how migrants subjectively experience the interaction. In previous work, we have shown that migrants' general cultural orientations, as well as characteristics of the social context, influence not only how people affiliate culturally in the moment, but also their affective reaction to the interaction (Doucerain, Dere, \& Ryder, 2013). This subjective reaction to the interaction, aggregated over time and across multiple interactions, contributes to adjust migrants' cultural orientations, updating in turn the likelihood and terms of future engagement.

This feedback process, frequently reiterated over many years, constitutes the macro-level third perspective. The present study only offers a snapshot of the beginning of the process, but the parallel unfolding of cultural orientations and social participation is likely to continue over time. Initial social participation with members of the mainstream cultural group did not predict changes in mainstream cultural orientations in this study, but this relation might emerge in later follow-ups, as migrants adjust their orientation in response to more long-term patterns of social interactions with members of the mainstream cultural group. Supporting this possibility, longitudinal studies focusing on initial stages of intergroup relations and intergroup contact show that the attitude to contact effect is at least as strong or stronger than the reverse effect (Eller et al., 2003; Levin et al., 2003). In contrast, examinations of intergroup relations that have been ongoing for a long time find stronger evidence for the contact to attitude effect (e.g., Binder et al., 2009; Levin et al., 2003). Accurately studying the development of this long-term reciprocal influence presents methodological difficulties as it would involve a high frequency of measurement over a very long time. A computational approach would address this issue. Agent-based computational models are increasingly used to examine cultural processes that do not lend themselves to field or laboratory studies (e.g., Dignum \& Dignum, 2013). Such an approach would be ideally suited to investigate the reciprocal influence between mainstream cultural orientations and mainstream social contact in the long term. It would also allow us to examine how the development of cultural knowledge ties into that dynamic.

This conceptual framing of the relation between cultural orientations and social participation is very tentative, but it allows us to consider (1) the significance of this association in the broader context of migrants' adaptation and (2) how to best push its exploration further. The present study documents one facet of this conceptual framing by establishing that initial cultural orientations toward the Canadian mainstream group prospectively predict early social participation in that group, above and beyond important alternative predictors. Two of its strengths further our confidence in the results. First, the longitudinal design allowed us to examine the specific role of initial cultural orientations and to test the hypothesized temporal direction of effects and its reverse. Second, in Study 2 great care was taken to recruit a sample with relatively homogeneous social functioning at baseline, with participants having no local social connections prior to migrating to the host country. Therefore, our results cannot be attributed to variations in social integration at baseline. 
However, reliance on a student sample limits the generalizability of results and introduces potential pressures on social participation. Students are more forced to interact with members of the mainstream cultural group and have more interaction opportunities in general compared to migrants in the community. Nonetheless, within these structural constraints, students make choices in navigating this social environment. The small size of our samples also constitutes a clear limitation of the two studies reported here. It is also important to note that our sample is culturally heterogeneous and that aspects of participants' cultural background may influence their social participation in the new environment. For example, a greater distance between the cultural heritage and the mainstream Canadian cultural tradition (itself a concept fraught with difficulties) or heritage cultural norms favoring more avoidance-oriented approaches in interpersonal relations, may make it more difficult or less desirable to socially participate in the new cultural environment. In future research, it would be important to examine how cultural characteristics of migrants' background play a role in the relation between cultural orientations and social participation. Meanwhile, the present studies address critiques that cultural orientations mostly reflect a general preference for multiculturalism and supports the idea that migrants' social engagement in the new community lies at the core of acculturation, in line with a dynamic intergroup process perspective on acculturation. We believe that this conceptual framing of acculturation is a promising direction for research on migrants' cultural adaptation.

\section{References}

Allport, G. W. (1954). The nature of prejudice. Cambridge, MA: Basic Books.

Amiot, C. E., de la Sablonniere, R., Terry, D. J., \& Smith, J. R. (2007). Integration of social identities in the self: Toward a cognitive-developmental model. Personality and Social Psychology Review, 11(4), 364-388. doi:10.1177/1088868307304091

Asendorpf, J. B., \& Wilpers, S. (1998). Personality effects on social relationships. Journal of Personality and Social Psychology, 74(6), 1531-1544. doi:10.1037/0022-3514.74.6.1531

Baumgarte, R. (2013). Friends beyond borders: Cultural variations in close friendship. North Charleston, SC: Createspace.

Berntson, G. G., Thomas Bigger, J., Eckberg, D. L., Grossman, P., Kaufmann, P. G., Malik, M., ... Van Der Molen, M. W. (1997). Heart rate variability: Origins, methods, and interpretive caveats. Psychophysiology, 34(6), 623-648. doi:10.1111/j.1469-8986.1997.tb02140.x

Berry, J. W. (2005). Acculturation: Living successfully in two cultures. International Journal of Intercultural Relations, 29(6), 697-712. doi:10.1016/j.ijintrel.2005.07.013

Binder, J., Zagefka, H., Brown, R., Funke, F., Kessler, T., Mummendey, A., ... \& Leyens, J. P. (2009). Does contact reduce prejudice or does prejudice reduce contact? A longitudinal test of the contact hypothesis among majority and minority groups in three European countries. Journal of personality and social psychology, 96(4), 843-756.

Bonnemeier, H., Wiegand, U. K., Brandes, A., Kluge, N., Katus, H. A., Richardt, G., \& Potratz, J. (2003). Circadian profile of cardiac autonomic nervous modulation in healthy subjects. Journal of Cardiovascular Electrophysiology, 14(8), 791799.

Boski, P. (2008). Five meanings of integration in acculturation research. International Journal of Intercultural Relations, 32(2), 142-153. doi:10.1016/j.ijintrel.2008.01.005

Bourhis, R. Y., Moïse, L. C., Perreault, S., \& Senécal, S. (1997). Towards an interactive acculturation model: A social psychological approach. International Journal of Psychology, 32(6), 369-386. doi:10.1080/002075997400629

Brown, R., Baysu, G., Cameron, L., Nigbur, D., Rutland, A., Watters, C., ... Landau, A. (2013). Acculturation attitudes and social adjustment in British South Asian children: A longitudinal study. Personality and Social Psychology Bulletin, 39(12), 1656-1667. doi:10.1177/0146167213500149

Brown, R., \& Zagefka, H. (2011). The dynamics of acculturation: An intergroup perspective. Advances in Experimental Social Psychology, 44, 129-184.

Cheek, J. M., \& Briggs, C. L. (1990). Shyness as a personality trait. In W. R. Crozier (Ed.), Shyness and embarrassment: Perspectives from social psychology (pp. 315-337). Cambridge, England: Cambridge University Press.

Chirkov, V. (2009). Critical psychology of acculturation: What do we study and how do we study it, when we investigate acculturation? International Journal of Intercultural Relations, 33(2), 94-105. doi:10.1016/j.ijintrel.2008.12.004

Chiswick, B. R., \& Miller, P. W. (1996). Ethnic networks and language proficiency among immigrants. Journal of Population Economics, 9(1), 19-35. doi:10.1007/PL00013277

Cohen, S., Doyle, W. J., Skoner, D. P., Rabin, B. S., \& Gwaltney, J. M. (1997). Social ties and susceptibility to the common cold. JAMA: The Journal of the American Medical Association, 277(24), 1940-1944.

Dignum, V., \& Dignum, F. (Eds.). (2013). Perspectives on culture and agent-based simulations: Integrating cultures. New York, NY: Springer. 
Doucerain, M., Dere, J., \& Ryder, A. G. (2013). Travels in hyper-diversity: Multiculturalism and the contextual assessment of acculturation. International Journal of Intercultural Relations, 37(6), 686-699. doi:10.1016/j.ijintrel.2013.09.007

Doucerain, M. M., Segalowitz, N., \& Ryder, A. G. (in press). Acculturation measurement: From simple proxies to sophisticated toolkit. In S. Schwartz, \& J. Unger (Eds.), Oxford, England: The Oxford Handbook of Acculturation and Health.

Eller, A., \& Abrams, D. (2003). 'Gringos' in Mexico: Cross-sectional and longitudinal effects of language school-promoted contact on intergroup bias. Group Processes \& Intergroup Relations, 6(1), 55-75.

Eller, A., \& Abrams, D. (2004). Come together: longitudinal comparisons of Pettigrew's reformulated intergroup contact model and the common ingroup identity model in Anglo-French and Mexican-American contexts. European Journal of Social Psychology, 34(3), 229-256.

Geisler, F. C. M., Kubiak, T., Siewert, K., \& Weber, H. (2013). Cardiac vagal tone is associated with social engagement and self-regulation. Biological Psychology, 93(2), 279-286. doi:10.1016/j.biopsycho.2013.02.013

Gyurak, A., \& Ayduk, O. (2008). Resting respiratory sinus arrhythmia buffers against rejection sensitivity via emotion control. Emotion, 8(4), 458-467. doi:10.1037/1528-3542.8.4.458

Hendrickson, B., Rosen, D., \& Aune, R. K. (2011). An analysis of friendship networks, social connectedness, homesickness, and satisfaction levels of international students. International Journal of Intercultural Relations, 35(3), $281-295$. doi:10.1016/j.ijintrel.2010.08.001

Hofstede, G. H. (2001). Culture's consequences: Comparing values, behaviors, institutions and organizations across nations. Thousand Oaks, CA: Sage.

Huynh, Q.-L., Howell, R. T., \& Benet-Martínez, V. (2009). Reliability of bidimensional acculturation scores: A metaanalysis. Journal of Cross-Cultural Psychology, 40(2), 256-274. doi:10.1177/0022022108328919

Kashima, Y. (2014). Meaning, grounding, and the construction of social reality. Asian Journal of Social Psychology, 17(2), 81-95. doi:10.1111/ajsp.12051

Kline, R. B. (2004). Beyond significance testing: Reforming data analysis methods in behavioral research. Washington, DC: APA.

Klusek, J., Martin, G. E., \& Losh, M. (2013). Physiological arousal in autism and fragile X syndrome: Group comparisons and links with pragmatic language. American Journal on Intellectual and Developmental Disabilities, 118(6), $475-495$. doi:10.1352/1944.7558-118.6.475

Levin, S., Van Laar, C., \& Sidanius, J. (2003). The effects of ingroup and outgroup friendships on ethnic attitudes in college: A longitudinal study. Group Processes \& Intergroup Relations, 6(1), 76-92.

Leys, C., Klein, O., Bernard, P., \& Licata, L. (2013). Detecting outliers: Do not use standard deviation around the mean, use absolute deviation around the median. Journal of Experimental Social Psychology, 49(4), 764-766. doi:10.1016/j.jesp.2013.03.013

Liebkind, K. (2001). Acculturation. In R. Brown \& S. Gaertner (Eds.), Blackwell handbook of social psychology: Intergroup processes (pp. 386-406). Oxford, UK: Blackwell.

Mähönen, T. A., \& Jasinskaja-Lahti, I. (2013). Acculturation expectations and experiences as predictors of ethnic migrants' psychological well-being. Journal of Cross-Cultural Psychology, 44(5), 786-806.

Martinovic, B., van Tubergen, F., \& Maas, I. (2011). Acquisition of cross-ethnic friends by recent immigrants in Canada: A longitudinal approach. International Migration Review, 45(2), 460-488.

Meyers, L. S., Gamst, G., \& Guarino, A. J. (2013). Applied multivariate research: Design and interpretation. Los Angeles, CA: Sage.

Nakagawa, S., \& Schielzeth, H. (2013). A general and simple method for obtaining $\mathrm{R}^{2}$ from generalized linear mixed-effects models. Methods in Ecology and Evolution, 4(2), 133-142. doi:10.1111/j.2041-210x.2012.00261.x

Nguyen, A.-M. D., \& Benet-Martínez, V. (2013). Biculturalism and adjustment: A meta-analysis. Journal of Cross-Cultural Psychology, 44(1), 122-159. doi:10.1177/0022022111435097

Nishida, H. (1999). A cognitive approach to intercultural communication based on schema theory. International Journal of Intercultural Relations, 23(5), 753-777. doi:10.1016/S0147-1767(99)00019-X

Pavlenko, A. (2004). Introduction: New theoretical approaches to the study of negotiation of identities in multilingual context. In A. Pavlenko \& A. Blackledge (Eds.), Negotiation of identities in multilingual contexts (pp. 1-33). Clevedon, England: Multilingual Matters.

Pettigrew, T. F., \& Tropp, L. R. (2006). A meta-analytic test of intergroup contact theory. Journal of Personality and Social Psychology, 90(5), 751-783.

Pinheiro, J. C., \& Bates, D. M. (2000). Mixed-effects models in S and S-PLUS. New York, NY: Springer.

Porges, S. W. (2007). The polyvagal perspective. Biological Psychology, 74(2), 116-143. doi:10.1016/j.biopsycho.2006.06.009

Porges, S. W., \& Bohrer, R. E. (1990). The analysis of periodic processes in psychophysiological research. In J. T. Cacioppo \& L. G. Tassinary (Eds.), Principles of psychophysiology: Physical, social, and inferential elements (pp. 708-753). New York, NY: Cambridge University Press.

Porter, C. L. (2003). Coregulation in mother-infant dyads: Links to infants' cardiac vagal tone. Psychological Reports, 92(1), 307-319. doi:10.2466/PR0.92.1.307-319

Ranney, S. (1992). Learning a new script: An exploration of sociolinguistic competence. Applied Linguistics, 13(1), 25-50. 
doi:10.1093/applin/13.1.25

Rudmin, F. (2009). Constructs, measurements and models of acculturation and acculturative stress. International Journal of Intercultural Relations, 33(2), 106-123. doi:10.1016/j.ijintrel.2008.12.001

Ryder, A. G., Alden, L. E., \& Paulhus, D. L. (2000). Is acculturation unidimensional or bidimensional? A head-to-head comparison in the prediction of personality, self-identity, and adjustment. Journal of Personality and Social Psychology, 79(1), 49-65. doi:10.1037/0022-3514.79.1.49

Sam, D. L., \& Berry, J. W. (2010). Acculturation: When individuals and groups of different cultural backgrounds meet. Perspectives on Psychological Science, 5(4), 472-481. doi:10.1177/1745691610373075

Sato, T. (2005). The Eysenck Personality Questionnaire Brief Version: Factor structure and reliability. The Journal of Psychology, 139(6), 545-552. doi:10.3200/JRLP.139.6.545-552

Schank, R. C., \& Abelson, R. P. (1977). Scripts, plans, goals and understanding: An inquiry into human knowledge structures. Hillsdale, NJ: Erlbaum.

Segalowitz, N. (2009). The Language Background Questionnaire. Unpublished instrument, Department of Psychology, Concordia University, Montreal, QC, Canada.

Segalowitz, N., Gatbonton, E., \& Trofimovich, P. (2009). Links between ethnolinguistic afiliation, self-rated motivation, and second language fluency. In Z. Dörnyei \& E. Ushioda (Eds.), Motivation, language identity and the L2 self (pp. 165171). Bristol, England: Multilingual Matters.

Simonsohn, U. (2014). Go big or go home: A practical alternative to impractical power analysis. Presented at the 15th annual meeting of the Society for Personality and Social Psychology, Austin, TX.

Singer, J. D., \& Willett, J. B. (2003). Applied longitudinal analysis: Modeling change and event occurrence. Oxford, England: Oxford University Press.

Smith, T. W., Cribbet, M. R., Nealey-Moore, J. B., Uchino, B. N., Williams, P. G., Mackenzie, J., \& Thayer, J. F. (2011). Matters of the variable heart: Respiratory sinus arrhythmia response to marital interaction and associations with marital quality. Journal of Personality and Social Psychology, 100(1), 103-119. doi:10.1037/a0021136

Stoessel, K., Titzmann, P.F., \& Silbereisen, R. K. (2012). Young diaspora immigrants' attitude and behavior toward the host culture: The role of cultural identification. European Psychologist, 17(2), 143-157. doi:10.1027/1016-9040/a000113

Tartakovsky, E. (2009). Cultural identities of adolescent immigrants: A three-year longitudinal study including the premigration period. Journal of Youth and Adolescence, 38(5), 654-671. doi:10.1007/s10964-008-9370-z

Titzmann, P. F., Silbereisen, R. K., \& Schmitt-Rodermund, E. (2007). Friendship homophily among diaspora migrant adolescents in Germany and Israel. European Psychologist, 12(3), 181-195. doi:10.1027/1016-9040.12.3.181

Van Oudenhoven, J. P., Ward, C., \& Masgoret, A. M. (2006). Patterns of relations between immigrants and host societies. International Journal of Intercultural Relations, 30(6), 637-651.

Wan, C., Dach-Gruschow, K., No, S., \& Hong, Y.(2010). Self-definitional functions of culture. In A. K. Leung, C. Y. Chiu, \& Y.Hong (Eds.), Cultural Processes: A Social Psychological Perspective (pp. 111-138). New York, NY:Cambridge University Press.

Williams, C. T., \& Johnson, L. R. (2011). Why can't we be friends?: Multicultural attitudes and friendships with international students. International Journal of Intercultural Relations, 35(1), 41-48. doi:10.1016/j.ijintrel.2010.11.001

Yip, T., \& Cross, W. E. (2004). A daily diary study of mental health and community involvement outcomes for three Chinese American social identities. Cultural Diversity \& Ethnic Minority Psychology, 10(4), 394-408. doi:10.1037/1099-9809.10.4.394

Zhang, J., \& Goodson, P. (2011). Predictors of international students' psychosocial adjustment to life in the United States: A systematic review. International Journal of Intercultural Relations, 35(2), 139-162. doi:10.1016/j.ijintrel.2010.11.011 